\title{
AN APPLICATION OF COHN'S RULE TO CONVOLUTIONS OF UNIVALENT HARMONIC MAPPINGS
}

\author{
RAJ KUMAR, SUSHMA GUPTA, SUKHJIT SINGH AND MICHAEL DORFF
}

\begin{abstract}
Dorff et al. [4] proved that the harmonic convolutions of the standard right half-plane mapping $F_{0}=$ $H_{0}+\bar{G}_{0}$ (where $H_{0}+G_{0}=z /(1-z)$ and $\left.G_{0}^{\prime}=-z H_{0}^{\prime}\right)$ and mappings $f_{\beta}=h_{\beta}+\bar{g}_{\beta}$ (where $f_{\beta}$ are obtained by shearing of analytic vertical strip mappings with dilatation $\left.e^{i \theta} z^{n}, n=1,2, \theta \in \mathbb{R}\right)$ are in $S_{H}^{0}$ and are convex in the direction of the real axis. In this paper, by using Cohn's rule, we generalize this result by replacing the standard right half-plane mapping $F_{0}$ with a family of right half-plane mappings $F_{a}=H_{a}+\bar{G}_{a}$ (with $H_{a}+G_{a}=z /(1-z)$ and $\left.G_{a}^{\prime} / H_{a}^{\prime}=(a-z) /(1-a z), a \in(-1,1)\right)$ and including the cases $n=3$ and $n=4$ (in addition to $n=1$ and $n=2$ ) for dilatations of $f_{\beta}$.
\end{abstract}

1. Introduction. Let $S_{H}$ denote the class of all harmonic, sensepreserving and univalent mappings $f=h+\bar{g}$ in the unit disk $E=$ $\{z:|z|<1\}$ which are normalized by the conditions $f(0)=0$ and $f_{z}(0)=1$. Therefore, a function $f=h+\bar{g}$ in the class $S_{H}$ has the representation,

$$
f(z)=z+\sum_{n=2}^{\infty} a_{n} z^{n}+\sum_{n=1}^{\infty} \bar{b}_{n} \bar{z}^{n},
$$

for all $z$ in $E$. We denote by $S_{H}^{0}$ the subclass of $S_{H}$ whose functions satisfy an additional condition of normalization $f_{\bar{z}}(0)=0$. Lewy [8] proved that a harmonic mapping $f=h+\bar{g}$ defined in $E$, is locally univalent and sense-preserving if and only if the Jacobian of the mapping, defined by $J_{f}=\left|h^{\prime}\right|^{2}-\left|g^{\prime}\right|^{2}$, is positive or, equivalently, if and only if

2010 AMS Mathematics subject classification. Primary 30C45.

Keywords and phrases. Cohn's rule, univalent harmonic mappings, harmonic convolutions.

The first author was supported by the Council of Scientific and Industrial Research, New Delhi, grant No. 09/797/0006/2010 EMR-1.

Received by the editors on July 3, 2013, and in revised form on June 11, 2014. 
$h^{\prime} \neq 0$ in $E$ and the dilatation function $\omega$ of $f$, defined by $\omega=g^{\prime} / h^{\prime}$, satisfies $|\omega(z)|<1$, for all $z \in E$. Further, let $K_{H}\left(K_{H}^{0}\right)$ be the subclass of $S_{H}\left(S_{H}^{0}\right)$ consisting of mappings which map $E$ onto convex domains. A domain $\Omega$ is said to be convex in the direction $\phi, 0 \leq \phi<\pi$, if every line parallel to the line joining 0 and $e^{i \phi}$ has a connected intersection with $\Omega$. For detailed and basic information about planar harmonic mappings one can refer to the monograph by Duren [5]. There are infinitely many harmonic mappings $F=H+\bar{G} \in S_{H}$ that map $E$ onto the right half-plane $\Psi=\{w: \Re(w)>-1 / 2\}$ and have the form $H+G=z /(1-z)$ with $\left|G^{\prime}(z)\right|<\left|H^{\prime}(z)\right|$ for all $z \in E$. Kumar et al. [7] defined a family of right half-plane harmonic mappings, $F_{a}=H_{a}+\bar{G}_{a}$, given by

$$
\begin{cases}H_{a}(z)+G_{a}(z)=\frac{z}{1-z}, & a \in(-1,1), \\ \frac{G_{a}^{\prime}(z)}{H_{a}^{\prime}(z)}=\frac{(a-z)}{1-a z}, & z \in E .\end{cases}
$$

By using the shear technique due to Clunie and Sheil-Small [1], we get

$$
H_{a}(z)=\frac{z /(1+a)-z^{2} / 2}{(1-z)^{2}} \quad \text { and } \quad G_{a}(z)=\frac{(a /(1+a) z)-z^{2} / 2}{(1-z)^{2}} .
$$

Setting $a=0$ in (1.2) above, we get the mapping $F_{0}=H_{0}+\bar{G}_{0}$ with $F_{0}+G_{0}=z /(1-z)$ and $G_{0}^{\prime}=-z H_{0}^{\prime}$, which is called the standard right half-plane mapping.

Let $\left\{f_{\beta}\right\}$, where $f_{\beta}=h_{\beta}+\bar{g}_{\beta}$, be the collection of harmonic mappings obtained by the shearing of analytic vertical strip mappings

$$
h_{\beta}(z)+g_{\beta}(z)=\frac{1}{2 i \sin \beta} \log \left(\frac{1+z e^{i \beta}}{1+z e^{-i \beta}}\right), \quad 0<\beta<\pi,
$$

with suitable dilatations. For more details about these mappings we refer the reader to Dorff [3].

The convolution, or Hadamard product, of two analytic functions

$$
h(z)=\sum_{n=1}^{\infty} a_{n} z^{n} \quad \text { and } \quad g(z)=\sum_{n=1}^{\infty} b_{n} z^{n}
$$


defined in $E$, is the function $h * g$ and is given by

$$
(h * g)(z)=\sum_{n=1}^{\infty} a_{n} b_{n} z^{n} .
$$

The function $h * g$ is also analytic in $E$. One can refer to Ruscheweyh and Sheil-Small [12] for more details. The harmonic convolution (or Hadamard product) of two harmonic mappings

$$
F(z)=H(z)+\bar{G}(z)=z+\sum_{n=2}^{\infty} A_{n} z^{n}+\sum_{n=1}^{\infty} \bar{B}_{n} \bar{z}^{n}
$$

and

$$
f(z)=h(z)+\bar{g}(z)=z+\sum_{n=2}^{\infty} a_{n} z^{n}+\sum_{n=1}^{\infty} \bar{b}_{n} \bar{z}^{n}
$$

in $S_{H}$ is defined as

$$
(F \widetilde{*} f)(z)=(H * h)(z)+\overline{(G * g)}(z)=z+\sum_{n=2}^{\infty} a_{n} A_{n} z^{n}+\sum_{n=1}^{\infty} \bar{b}_{n} \bar{B}_{n} \bar{z}^{n} .
$$

It is well known (for example, see [2]) that, unlike the case of conformal mappings, harmonic convolution of two mappings from the class $K_{H}^{0}$ may not be in $K_{H}^{0}$ and may not even be in $S_{H}^{0}$. Also, if $f_{1} \in K_{H}^{0}$ and $f_{2} \in S_{H}^{0}$, then $f_{1} \widetilde{*} f_{2}$ is not necessarily in $S_{H}^{0}$. These facts generated a lot of interest in the study of harmonic convolutions of univalent harmonic mappings, and a good number of papers recently appeared in the literature on this topic (for example, see $[\mathbf{2}, \mathbf{4}, \mathbf{6}, \mathbf{7}$, 9, 10]). In particular, Dorff [2] proved the following result:

Theorem A. Let $F=H+\bar{G}$ with $H+G=z /(1-z)$ be any right halfplane mapping, and let $f_{\beta}=h_{\beta}+\bar{g}_{\beta} \in K_{H}^{0}$ be given by equation (1.3) with $\pi / 2 \leq \beta<\pi$. Then $F \widetilde{*} f_{\beta} \in S_{H}^{0}$ and is convex in the direction of the real axis, provided $F \widetilde{*} f_{\beta}$ is locally univalent and sense-preserving.

Recently, Dorff et al. [4] obtained the following result which shows that the condition of harmonic convolution being locally univalent and sense-preserving, as required in Theorem A, can be dropped in some special cases but not in general. 
Theorem B. If $F_{0}=H_{0}+\bar{G}_{0}$ is the standard right half-plane mapping and $f_{\beta}=h_{\beta}+\bar{g}_{\beta}$ is the harmonic mapping as defined in Theorem $\mathrm{A}$ above, with dilatation $\omega_{n}(z)=g_{\beta}^{\prime} / h_{\beta}^{\prime}=e^{i \theta} z^{n}$, then, for $n=1,2$, $F_{0} \widetilde{*} f_{\beta} \in S_{H}^{0}$ and is convex in the direction of the real axis.

Proceeding as in [4, Remark 1], one can verify that Theorem B does not hold true for $n \geq 3$. The aim of the present paper is to study harmonic convolutions of more general harmonic mappings $F_{a}=H_{a}+\bar{G}_{a}$, where $H_{a}$ and $G_{a}$ are defined by (1.2) and the mappings $f_{\beta}=h_{\beta}+\bar{g}_{\beta}$, where $h_{\beta}$ and $g_{\beta}$ are the solutions of (1.3), with dilatation $\omega_{n}(z)=g_{\beta}^{\prime} / h_{\beta}^{\prime}=e^{i \theta} z^{n}, n \in \mathbb{N}$. We shall find the range of the real constant $a$ such that $F_{a} \widetilde{*} f_{\beta} \in S_{H}^{0}$ and convex in the direction of the real axis, even if $n \geq 3$. Cohn's rule, stated below, shall play a central role in the proofs of our results in this paper.

Lemma A. (Cohn's rule [11, page 375]). Given a polynomial

$$
t(z)=a_{0}+a_{1} z+a_{2} z^{2}+\cdots+a_{n} z^{n}
$$

of degree $n$, let

$$
t^{*}(z)=z^{n} \overline{t\left(\frac{1}{\bar{z}}\right)}=\bar{a}_{n}+\bar{a}_{n-1} z+\bar{a}_{n-2} z^{2}+\cdots+\bar{a}_{0} z^{n} .
$$

Denote by $r$ and $s$ the number of zeros of $t$ inside and on the unit circle $|z|=1$, respectively. If $\left|a_{0}\right|<\left|a_{n}\right|$, then

$$
t_{1}(z)=\frac{\bar{a}_{n} t(z)-a_{0} t^{*}(z)}{z}
$$

is of degree $n-1$ and has $r_{1}=r-1$ and $s_{1}=s$ number of zeros inside and on the unit circle $|z|=1$, respectively.

2. Main results. In addition to Cohn's rule stated above, we begin by proving the following two results which are also essential for the proofs of our main results.

Lemma 2.1. Let $f_{\beta}=h_{\beta}+\bar{g}_{\beta} \in K_{H}^{0}$ be given by equation (1.3) with dilatation $\omega=g_{\beta}^{\prime} / h_{\beta}^{\prime}$, and let $F_{a}=H_{a}+\bar{G}_{a}$ be the right half-plane 
mapping defined by (1.2). Then $\widetilde{\omega}$, the dilatation of $F_{a} \widetilde{*} f_{\beta}$, is given by (2.1) $\widetilde{\omega}(z)=\left[\frac{2 \omega(1+\omega)\left(a+a z \cos \beta+z \cos \beta+z^{2}\right)-z \omega^{\prime}(1-a)\left(1+2 z \cos \beta+z^{2}\right)}{2\left(1+z \cos \beta+a z \cos \beta+a z^{2}\right)(1+\omega)-z \omega^{\prime}(1-a)\left(1+2 z \cos \beta+z^{2}\right)}\right]$.

Proof. Since

$$
F_{a} \widetilde{*} f_{\beta}=H_{a} * h_{\beta}+\overline{G_{a} * g_{\beta}}=H+\bar{G}
$$

(say), so

$$
H(z)=\frac{1}{2}\left[h_{\beta}+\frac{(1-a)}{(1+a)} z h_{\beta}^{\prime}\right]
$$

and

$$
G(z)=\frac{1}{2}\left[g_{\beta}-\frac{(1-a)}{(1+a)} z g_{\beta}^{\prime}\right]
$$

If $\widetilde{\omega}$ is the dilatation of $F_{a} \widetilde{*} f_{\beta}$, then

$$
\widetilde{\omega}(z)=\frac{G^{\prime}(z)}{H^{\prime}(z)}=\left[\frac{2 a g_{\beta}^{\prime}-(1-a) z g_{\beta}^{\prime \prime}}{2 h_{\beta}^{\prime}+(1-a) z h_{\beta}^{\prime \prime}}\right] .
$$

Since $g_{\beta}^{\prime}=\omega h_{\beta}^{\prime}$,

$$
h_{\beta}^{\prime}(z)=\frac{1}{(1+\omega)\left(1+z e^{i \beta}\right)\left(1+z e^{-i \beta}\right)}
$$

and

$$
h_{\beta}^{\prime \prime}(z)=-\frac{2(\cos \beta+z)(1+\omega)+\omega^{\prime}\left(1+2 z \cos \beta+z^{2}\right)}{(1+\omega)^{2}\left(1+z e^{i \beta}\right)^{2}\left(1+z e^{-i \beta}\right)^{2}} ;
$$

therefore, from (2.2), we get

$$
\widetilde{\omega}(z)=\left[\frac{2 a \omega h_{\beta}^{\prime}-(1-a) z\left(\omega h_{\beta}^{\prime \prime}+\omega^{\prime} h_{\beta}^{\prime}\right)}{2 h_{\beta}^{\prime}+(1-a) z h_{\beta}^{\prime \prime}}\right] .
$$

Substituting the values of $h_{\beta}^{\prime}$ and $h_{\beta}^{\prime \prime}$ and simplifying, we get (2.1).

Lemma 2.2. The following inequalities hold true:

(a) $\left|-2 \cos \beta+4 e^{-i \theta} \cos ^{2} \beta-3 e^{-i \theta}+e^{i \theta}\right| \leq\left|4-2 \cos ^{2} \beta-2 \cos \beta \cos \theta\right|$ for $\beta \in(0, \pi)$ and $\theta \in \mathbb{R}$.

(b) $\left|\cos \beta\left(e^{-i \theta}-5\right)\right|<\left|6-\cos ^{2} \beta+2 e^{-i \theta}-3 e^{-i \theta} \cos ^{2} \beta\right|$ for $\beta \in(0, \pi)$ and $\theta \in \mathbb{R}$. 
(c) $\left|2\left(1+e^{-i \theta}\right)-3 e^{-i \theta} \cos ^{2} \beta\right|<\left|4-\cos ^{2} \beta\right|$ for $\beta \in(0, \pi / 2) \cup(\pi / 2, \pi)$ and $\theta \neq 2 m \pi, m \in \mathbb{Z}$.

Proof. Let $x=\cos \beta$ and $y=\cos \theta$. Then

(a)

$$
\begin{aligned}
& \left|-2 \cos \beta+4 e^{-i \theta} \cos ^{2} \beta-3 e^{-i \theta}+e^{i \theta}\right|^{2}-\left|4-2 \cos ^{2} \beta-2 \cos \beta \cos \theta\right|^{2} \\
& =12 x^{4}-12 x^{2}-24 x^{3} y+24 x y+12 x^{2} y^{2}-12 y^{2} \\
& =-12\left(1-x^{2}\right)(x-y)^{2} \leq 0 \\
& \quad \text { as } x \in(-1,1) \text { and } y \in[-1,1] .
\end{aligned}
$$

(b)

$$
\begin{gathered}
\left|\cos \beta\left(e^{-i \theta}-5\right)\right|^{2}-\left|6-\cos ^{2} \beta+2 e^{-i \theta}-3 e^{-i \theta} \cos ^{2} \beta\right|^{2} \\
=-2\left(5 x^{4}+3 x^{4} y-15 x^{2} y-25 x^{2}+12 y+20\right) \\
=-2\left(x^{2}-4\right)\left(x^{2}-1\right)(5+3 y)<0, \\
\quad \text { for } x \in(-1,1) \text { and } y \in[-1,1] .
\end{gathered}
$$

(c)

$$
\begin{aligned}
& \left|2\left(1+e^{-i \theta}\right)-3 e^{-i \theta} \cos ^{2} \beta\right|^{2}-\left|4-\cos ^{2} \beta\right|^{2} \\
& \quad=4\left(2 x^{4}-x^{2}-3 y x^{2}+2 y-2\right) \\
& \quad=4\left[2\left(x^{2}-1\right)\left(x^{2}+1-y\right)-x^{2}(y+1)\right]<0, \\
& \quad \text { for } x \in(-1,0) \cup(0,1) \text { and } y \in[-1,1) .
\end{aligned}
$$

We are now in a position to prove our main results.

Theorem 2.3. Let $f_{\beta}=h_{\beta}+\bar{g}_{\beta} \in K_{H}^{0}$ be given by (1.3) with dilatation $\omega_{1}(z)=e^{i \theta} z$. If $F_{a}$ is the right half-plane mapping defined by (1.2), then $F_{a} \widetilde{*} f_{\beta} \in S_{H}^{0}$ and is convex in the direction of the real axis for $a \in[-1 / 3,1)$.

Proof. Let $\widetilde{\omega}$ be the dilatation of $F_{a} \widetilde{*} f_{\beta}$. We claim that $|\widetilde{\omega}(z)|<1$ for all $z \in E$, i.e., $F_{a} \widetilde{*} f_{\beta}$ is locally univalent and sense-preserving in $E$. Our result shall then follow from Theorem A. By setting 
$\omega(z)=\omega_{1}(z)=e^{i \theta} z$ in $(2.1)$, we get

$$
\begin{gathered}
\widetilde{\omega}(z) \\
=z\left[\frac{e^{i \theta} z^{3}+\left(\frac{1}{2}+a e^{i \theta} \cos \beta+e^{i \theta} \cos \beta+a / 2\right) z^{2}+a\left(2 \cos \beta+e^{i \theta}\right) z+\frac{3 a-1}{2}}{e^{-i \theta}+\left(e^{-i \theta} \cos \beta+a e^{-i \theta} \cos \beta+\frac{1}{2}+\frac{a}{2}\right) z+a\left(e^{-i \theta}+2 \cos \beta\right) z^{2}+\frac{3 a-1}{2} z^{3}}\right] \\
=z \frac{p(z)}{p^{*}(z)},
\end{gathered}
$$

where

$$
\begin{aligned}
p(z)= & e^{i \theta} z^{3}+\left(\frac{1}{2}+a e^{i \theta} \cos \beta+e^{i \theta} \cos \beta+\frac{a}{2}\right) z^{2} \\
& +a\left(2 \cos \beta+e^{i \theta}\right) z+\frac{3 a-1}{2} \\
= & a_{3} z^{3}+a_{2} z^{2}+a_{1} z+a_{0}
\end{aligned}
$$

and

$$
\begin{aligned}
p^{*}(z)= & e^{-i \theta}+\left(e^{-i \theta} \cos \beta+a e^{-i \theta} \cos \beta+\frac{1}{2}+\frac{a}{2}\right) z \\
& +a\left(e^{-i \theta}+2 \cos \beta\right) z^{2}+\frac{3 a-1}{2} z^{3}=z^{3} p \overline{\left(\frac{1}{\bar{z}}\right)} .
\end{aligned}
$$

Thus, if $z_{0}, z_{0} \neq 0$, is a zero of $p$, then $1 / \bar{z}_{0}$ is a zero of $p^{*}$. Therefore, we can write

$$
\widetilde{\omega}(z)=z \frac{(z+A)(z+B)(z+C)}{(1+\bar{A} z)(1+\bar{B} z)(1+\bar{C} z)} .
$$

In order to prove that $|\widetilde{\omega}(z)|<1$ in $E$, it suffices to show that $|A| \leq 1$, $|B| \leq 1$ and $|C| \leq 1$, or equivalently, all the zeros, $-A,-B$ and $-C$, of the polynomial $p$ lie in or on the unit circle $|z|=1$ for $a \in[-1 / 3,1)$. When $a=-1 / 3$, then $|\widetilde{\omega}(z)|=\left|-z e^{i \theta}\right|<1$ for all $z \in E$, and we take $a \in(-1 / 3,1 / 3) \cup(1 / 3,1)$, so the case for $a=1 / 3$ will be settled separately.

Let 


$$
\begin{aligned}
p_{1}(z) & =\frac{\bar{a}_{3} p(z)-a_{0} p^{*}(z)}{z} \\
(2.3) & =\frac{1}{4}\left(1+2 a-3 a^{2}\right)\left[3 z^{2}+2\left(2 \cos \beta+e^{-i \theta}\right) z+\left(2 \cos \beta e^{-i \theta}+1\right)\right] \\
& =b_{2} z^{2}+b_{1} z+b_{0} .
\end{aligned}
$$

As $\left|a_{0}\right|<\left|a_{3}\right|$ and $1+2 a-3 a^{2} \neq 0$ for $a \in(-1 / 3,1 / 3) \cup(1 / 3,1)$; therefore, by Cohn's rule, the polynomial $p_{1}$ has one less number of zeros inside $|z|=1$ than $p$ and the same number of zeros on $|z|=1$ as p. Again, let

$$
\begin{aligned}
p_{2}(z)= & \frac{\bar{b}_{2} p_{1}(z)-b_{0} p_{1}^{*}(z)}{z} \\
= & \frac{1}{16}\left(1+2 a-3 a^{2}\right)^{2}\left[\left(9-\left|2 \cos \beta+e^{i \theta}\right|^{2}\right) z\right. \\
& \left.+6\left(2 \cos \beta+e^{-i \theta}\right)-2 e^{-i \theta}\left(2 \cos \beta+e^{i \theta}\right)^{2}\right]
\end{aligned}
$$

where $p_{1}^{*}(z)=z^{2} \overline{p_{1}(1 / \bar{z})}$. We can again apply Cohn's rule on $p_{1}$ because

$$
\left|b_{0}\right|=\left|\left(1+2 a-3 a^{2}\right) \frac{1}{4}\left(2 \cos \beta e^{-i \theta}+1\right)\right|<\left|\left(1+2 a-3 a^{2}\right) \frac{3}{4}\right|=\left|b_{2}\right| .
$$

If the zero of $p_{2}$ is denoted by $z_{0}$, then

$$
z_{0}=\frac{-2 \cos \beta+4 e^{-i \theta} \cos ^{2} \beta-3 e^{-i \theta}+e^{i \theta}}{4-2 \cos ^{2} \beta-2 \cos \beta \cos \theta}
$$

and in view of Lemma 2.2 (a), $\left|z_{0}\right| \leq 1$. Thus, by Cohn's rule, all the zeros of $p_{1}$ and therefore of $p$ lie inside or on the unit circle $|z|=1$ for $a \in(-1 / 3,1 / 3) \cup(1 / 3,1)$. In the case when $a=1 / 3$, then

$$
p(z)=\frac{1}{3} e^{i \theta} z\left[3 z^{2}+2\left(2 \cos \beta+e^{-i \theta}\right) z+\left(2 \cos \beta e^{-i \theta}+1\right)\right] .
$$

In view of (2.3), it is easy to verify that all the zeros of $p$ lie inside or on the circle $|z|=1$ for $a=1 / 3$. This completes the proof.

Remark 2.4. By setting $a=0$ in Theorem 2.3, we get Theorem B (for $n=1$ ), stated in Section 1. 
Theorem 2.5. If $f_{\beta}$ is given by equation (1.3) with dilatation $\omega_{2}(z)=$ $e^{i \theta} z^{2}$, then $F_{a} \widetilde{*} f_{\beta} \in S_{H}^{0}$ and is convex in the direction of the real axis for $a \in[0,1)$, where $F_{a}$ is defined by equation (1.2).

Proof. The case when $a=0$ has already been proved by Dorff et al. [4], so we take $a \in(0,1)$. Now, if $\widetilde{\omega}$ is the dilatation of $F_{a} \widetilde{*} f_{\beta}$, then letting $\omega(z)=\omega_{2}(z)=e^{i \theta} z^{2}$ in equation (2.1), we get

$$
\begin{aligned}
& \widetilde{\omega}(z)=z^{2} e^{2 i \theta} \\
& \cdot\left[\frac{z^{4}+\cos \beta(a+1) z^{3}+a\left(1+e^{-i \theta}\right) z^{2}+e^{-i \theta} \cos \beta(3 a-1) z+e^{-i \theta}(2 a-1)}{1+\cos \beta(a+1) z+a\left(1+e^{i \theta}\right) z^{2}+e^{i \theta} \cos \beta(3 a-1) z^{3}+e^{i \theta}(2 a-1) z^{4}}\right] .
\end{aligned}
$$

In view of Theorem $\mathrm{A}$, we need only show that $F_{a} \widetilde{*} f_{\beta}$ is locally univalent and sense-preserving in $E$, i.e., $|\widetilde{\omega}|<1$ in $E$. Consider

$$
\begin{aligned}
q(z)= & z^{4}+\cos \beta(a+1) z^{3}+a\left(1+e^{-i \theta}\right) z^{2} \\
& +e^{-i \theta} \cos \beta(3 a-1) z+e^{-i \theta}(2 a-1) \\
= & a_{4} z^{4}+a_{3} z^{3}+a_{2} z^{2}+a_{1} z+a_{0},
\end{aligned}
$$

and

$$
\begin{aligned}
q^{*}(z)= & z^{4} \overline{q(1 / \bar{z})} \\
= & 1+\cos \beta(a+1) z+a\left(1+e^{i \theta}\right) z^{2} \\
& +e^{i \theta} \cos \beta(3 a-1) z^{3}+e^{i \theta}(2 a-1) z^{4} .
\end{aligned}
$$

Then

$$
\widetilde{\omega}(z)=z^{2} e^{2 i \theta} \frac{q(z)}{q^{*}(z)}=z^{2} e^{2 i \theta} \frac{(z+A)(z+B)(z+C)(z+D)}{(1+\bar{A} z)(1+\bar{B} z)(1+\bar{C} z)(1+\bar{D} z)},
$$

where $-A,-B,-C$ and $-D$ are the zeros of $q$. We shall show that these zeros lie inside or on the unit circle $|z|=1$ for $a \in(0,1)$. First, we take $a \in(0,1 / 2) \cup(1 / 2,1)$, and the case when $a=1 / 2$ will be dealt with separately. For $a \in(0,1 / 2) \cup(1 / 2,1)$, we have $\left|a_{0}\right|=|2 a-1|<1=\left|a_{4}\right|$, so we can apply Cohn's rule on $q$. Let

$$
\begin{aligned}
q_{1}(z) & =\frac{\bar{a}_{4} q(z)-a_{0} q^{*}(z)}{z} \\
& =2 a(1-a)\left(2 z^{3}+3 \cos \beta z^{2}+\left(1+e^{-i \theta}\right) z+e^{-i \theta} \cos \beta\right) \\
& =b_{3} z^{3}+b_{2} z^{2}+b_{1} z+b_{0} .
\end{aligned}
$$


It is easy to verify that Cohn's rule is applicable to $q_{1}$ also. So, let

$$
\begin{aligned}
q_{2}(z)= & \frac{\bar{b}_{3} q_{1}(z)-b_{0} q_{1}^{*}(z)}{z} \\
= & 4(a(1-a))^{2}\left[\left(4-\cos ^{2} \beta\right) z^{2}+\cos \beta\left(5-e^{-i \theta}\right) z\right. \\
& \left.\quad+2\left(1+e^{-i \theta}\right)-3 e^{-i \theta} \cos ^{2} \beta\right] \\
= & c_{2} z^{2}+c_{1} z+c_{0} .
\end{aligned}
$$

To apply Cohn's rule again on $q_{2}$ we need

$$
\left|2\left(1+e^{-i \theta}\right)-3 e^{-i \theta} \cos ^{2} \beta\right|<\left|4-\cos ^{2} \beta\right|,
$$

which is true in view of Lemma 2.2 (c), provided $\beta \neq \pi / 2$ and $\theta \neq 2 m \pi$, $m \in \mathbb{Z}$. Therefore, by applying Cohn's rule again on $q_{2}$, we have

$$
\begin{aligned}
q_{3}(z)= & \frac{\bar{c}_{2} q_{2}(z)-c_{0} q_{2}^{*}(z)}{z} \\
= & 16(a(1-a))^{4}\left\{\left[\left(4-\cos ^{2} \beta\right)^{2}-\left(2\left(1+e^{-i \theta}\right)-3 e^{-i \theta} \cos ^{2} \beta\right)^{2}\right] z\right. \\
& \left.+\cos \beta\left(5-e^{-i \theta}\right)\left[\left(4-\cos ^{2} \beta\right)-2\left(1+e^{-i \theta}\right)+3 e^{-i \theta} \cos ^{2} \beta\right]\right\} .
\end{aligned}
$$

The only zero of $q_{3}$ shall lie inside or on the circle $|z|=1$, provided

$$
\left|\cos \beta\left(e^{-i \theta}-5\right)\right|<\left|6-\cos ^{2} \beta+2 e^{-i \theta}-3 e^{-i \theta} \cos ^{2} \beta\right|,
$$

which is true in view of Lemma 2.2 (b). Thus, by Cohn's rule, all zeros of $q_{2}$ and $q_{1}$, and therefore of $q$, lie in or on the unit circle $|z|=1$ for $\beta \neq \pi / 2$ and $\theta \neq 2 m \pi, m \in \mathbb{Z}$.

In the cases where $\beta=\pi / 2$ and $\theta=2 m \pi, m \in \mathbb{Z}$, we have

$$
q_{2}(z)=16(a(1-a))^{2}\left(z^{2}+1\right),
$$

and, obviously, zeros of $q_{2}$ lie on $|z|=1$ because $a \in(0,1 / 2) \cup(1 / 2,1)$. Therefore, by Cohn's rule, all zeros of $q_{1}$ and of $q$ lie in or on the unit circle $|z|=1$ in this case also.

Now when $a=1 / 2$, then we have

$$
q(z)=\frac{z}{2}\left(2 z^{3}+3 \cos \beta z^{2}+\left(1+e^{-i \theta}\right) z+e^{-i \theta} \cos \beta\right) .
$$

Keeping (7) in mind, we can easily verify that, in this case, all zeros of $q$ lie in or on the unit circle $|z|=1$. Hence, $|\widetilde{\omega}|<1$ for $a \in[0,1)$. 
As pointed out in Section 1, Theorem B is not true for $n \geq 3$; however, the following theorem, which we state without proof (as the proof runs on the same lines as of Theorem 2.5), asserts that the conclusion of Theorem 2.5 remains valid when dilatation of $f_{\beta}$ is taken as $e^{i \theta} z^{n}, n=3,4$, provided the value of the real constant $a$ is suitably restricted.

Theorem 2.6. Let $F_{a}$ be given by (1.2). Then we have the following:

(a) if $f_{\beta}$ is given by (1.3) with dilatation $\omega_{3}(z)=e^{i \theta} z^{3}$, then $F_{a} \widetilde{*} f_{\beta} \in$ $S_{H}^{0}$ and is convex in the direction of the real axis for $a \in[1 / 5,1)$;

(b) if $f_{\beta}$ is given by (1.3) with dilatation $\omega_{4}(z)=e^{i \theta} z^{4}$, then $F_{a} \widetilde{*} f_{\beta} \in$ $S_{H}^{0}$ and is convex in the direction of the real axis for $a \in[1 / 3,1)$.

Remark 2.7. It is expected that, keeping $\omega_{n}(z)=e^{i \theta} z^{n}(\theta \in \mathbb{R}, n \in \mathbb{N})$ as the dilatation of $f_{\beta}$, the convolution $F_{a} \widetilde{*} f_{\beta} \in S_{H}^{0}$ and is convex in the direction of the real axis for $a \in[(n-2) /(n+2), 1)$. We observe that, in the cases when $n \geq 5$, calculations become extremely cumbersome when we follow the above method of proof.

Acknowledgments. The first author is thankful to the Council of Scientific and Industrial Research, New Delhi, for financial support. The authors are thankful to the learned referee for his valuable comments and suggestions.

\section{REFERENCES}

1. J. Clunie and T. Sheil-Small, Harmonic univalent functions, Ann. Acad. Sci. Fenn. Math. 9 (1984), 3-25.

2. M. Dorff, Convolutions of planar harmonic convex mappings, Comp. Var. Theor. Appl. 45 (2001), 263-271.

3. _ Harmonic mappings onto asymmetric vertical strips, in Computational methods and function theory, N. Papamichael, St. Ruscheweyh and E.B. Saff, eds., World Scientific Publishing, River Edge, NJ, 1999.

4. M. Dorff, M. Nowak and M. Woloszkiewicz, Convolutions of harmonic convex mappings, Comp. Var. Ellip. Eqn. 57 (2012), 489-503.

5. P.L. Duren, Harmonic mappings in the plane, Cambridge University Press, Cambridge, 2004.

6. M. Goodloe, Hadamard products of harmonic mappings, Comp. Var. Theor. Appl. 47 (2002), 81-92. 
7. R. Kumar, M. Dorff, S.Gupta and S.Singh, Convolution properties of some harmonic mappings in the right half-plane, Bull. Malays. Math. Sci. Soc. 39 (2016), $439-455$.

8. H. Lewy, On the non vanishing of the jacobian in certain one to one mappings, Bull. Amer. Math. Soc. 42 (1936), 689-692.

9. L. Li and S. Ponnusamy, Solution to an open problem on convolutions of harmonic mappings, Comp. Var. Ellip. Eqn. 58 (2013), 1647-1653.

10. , Convolution of slanted right half-plane harmonic mappings, Analy. Int. J Anal. Appl. 32 (2013), 159-176.

11. Q.I. Rahman and G. Schmeisser, Analytic theory of polynomials, Lond. Math. Soc. Mono. 26, Oxford University Press, Oxford, 2002.

12. St. Ruscheweyh and T. Sheil-Small, Hadamard products of schlicht functions and the Polya-Schoenberg conjecture, Comment Math Helv. 48 (1973), 119-135.

DAV University, Jalandhar-144001(Punjab), India

Email address: rajgarg2012@yahoo.co.in

Sant Longowal Institute of Engineering and Technology, Longowal148106 (Punjab), India

Email address: sushmagupta1@yahoo.com

Sant Longowal Institute of Engineering and Technology, Longowal148106 (PunJab), India

Email address: sukhjit_d@yahoo.com

Department of Mathematics, Brigham Young University, Provo, Utah, 84602

Email address: mdorff@math.byu.edu 